\title{
Preliminary Results of an Instrument for Measuring the Optical Aberrations of the Human Eye
}

\author{
Luis Alberto V. de Carvalho and Jarbas Caiado de Castro \\ Laboratório de Óptica Oftálmica - Grupo de Óptica, IFSC-USP \\ São Carlos, SP, CEP 13560-900, Brazil
}

Received on 31 August, 2002

\begin{abstract}
The human eye, as our biological vision instrument, contains intrinsic optical defects, referred to as optical aberrations or ametropia. The immediate consequence of such aberrations is poor quality of images formed at the retina. With the advent of more precise pulsed lasers for eye surgery, the development of instrumentation to determine precisely the higher order aberrations of the eye became a crucial chalange. Current instruments available commercially (refractometers) measure only the lower optical aberrations of the eye, i. e., myopia, hyperopia and astigmatism. In the present work we have developed a high resolution refractometer based on the Hartmann-Shack (HS) wave-front sensor. The HS sensor was originally developed for aberration measurements in general optical systems, and is of wide-spread usage in adaptive optics applications such as astronomical telescopes. Preliminary results for a mechanical eye are presented here and the RMSE in dioptric power (D) and cylinder axis (in degrees) were as follows: $0.04 \mathrm{D}$ for sphere and cylinder and $4^{0}$ for axis. It is known that refractometers have typical errors of $0.12 \mathrm{D}$ diopters for sphere and cylinder and $5^{0}$ for axis. These preliminary results indicate that the HS sensor may be applied successfully on in vivo eyes and that, in the near future, this technology may be available in most eye hospitals and clinics throughout the world, therefore bringing benefits to the general population.
\end{abstract}

\section{Introduction}

\section{I.1 Aberration theory}

The principle of image formation through optical systems is far from being a trivial subject. There are many variations in optical materials, surface curvatures and tolerances, distances between elements, quantity and function of each optical element, and so on. In the paraxial approximation the image formation by spherical refracting surfaces are only approximately correct. In devising ray-tracing formulas for these surfaces it is necessary to assume that rays form small angles with the refracting surface and also strike the surface close to the optical axis. In paraxial optics, the power expansions of sine and cosine, given by

$$
\sin x=x-\frac{x^{3}}{3 !}+\frac{x^{5}}{5 !}-\ldots, \cos x=1-\frac{x^{2}}{2 !}+\frac{x^{4}}{4 !}-\ldots
$$

are approximated by their first terms. To the extent that these first order approximations are valid, Gaussian Optics implies exact imaging. The inclusion of higher order terms in the derivations, however, predicts increasingly higher departures from "perfect imaging". These departures are referred to as optical aberrations. In order to quantify how well an optical system can transfer objects from object space to the image space, elaborate theories of optical aberration have been developed [1]. An illustration for a brief definition of optical aberration may be seen in Fig. 1.

Figure 1 shows a diagram of the image formation by a refracting surface separating two media of different refractive indexes $\left(n\right.$ and $\left.n^{\prime}\right)$. An object point at position $(p)$ localized at the object plane $(O)$ has it's image formed at point $\left(p^{\prime}\right)$ on the image plane $(I)$. A marginal ray intersects the exit pupil at some point $(\rho, \theta)$ of the surface. The wave aberration $(W)$ along the marginal ray is calculated as the difference in optical path length (OPL) from the chief ray, i.e.,;

$$
W=O P L_{\text {marginal }}-O P L_{\text {chief }}
$$

Using ray-tracing techniques and Snell's Law, if the surface position and shape is known (such as in cylindrical coordinates, i. e., $z=z(\rho, \theta)$ ) and also the refracting indexes of the different media, for a certain object point one may calculate the wave-aberration function for each ray pencil passing through the exit pupil. For the illustration in Fig. 1 the wave aberration for the considered ray is:

$$
W=n d+n^{\prime} d^{\prime}-n l-n^{\prime} l^{\prime}
$$




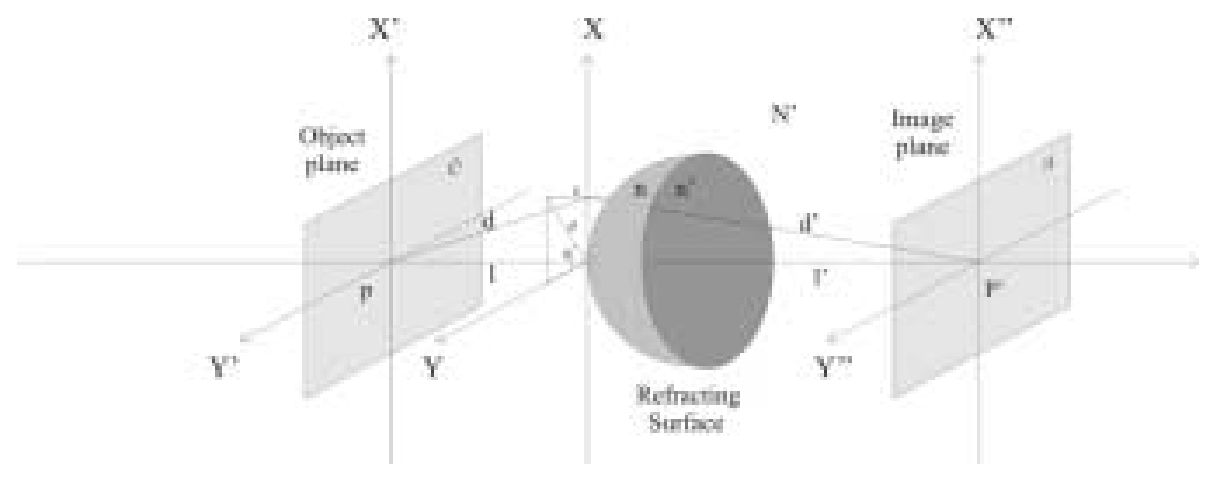

Figure 1. Definition of wave-front aberration for a refracting surface.

When the third order terms from the cosine and sine expansions in equation (1) are included, third-order aberration theory results. These aberrations have been studied and formalized in 1856 by the German mathematician Ludwig Von Seidel and are referred to as Seidel Aberrations. The Seidel Aberrations may be represented by a power series in polar coordinates $(\rho, \theta)$ :

$$
W(\rho, \theta)=\sum_{n, m=0}^{\infty} S_{n l 1} \rho^{n} \cos ^{l} \theta+S_{n l 2} \rho^{n} \sin ^{l} \theta
$$

In this series the Seidel Aberrations, a total of six, are explicit: piston, tilt, curvature of field, astigmatism, coma, spherical aberration (please refer to Table 1). An additional aberration, called chromatic aberration, results from the dispersion curve (refractive index variation with wave-length) of certain optical materials.

\begin{tabular}{llll}
\hline $\mathrm{n}$ & 1 & Representation (polar) & Description \\
\hline 0 & 0 & 1 & Piston \\
1 & 1 & $\rho \cos \theta$ & Tilt \\
2 & 0 & $\rho^{2}$ & Curvature of Field \\
2 & 2 & $\rho^{2} \cos ^{2} \theta$ & Astigmatism \\
3 & 1 & $\rho^{3} \cos \theta$ & Coma \\
4 & 0 & $\rho^{4}$ & Spherical Aberration
\end{tabular}

Table 1. Seidel Aberrations.

Because circular apertures are very common in optical systems, such as in telescopes, lenses and also the human eye (for example, the crystalline lens and eye pupil), the treatment in polar coordinates is very attractive. The power series that describes the Seidel Aberrations are, unfortunately, not an orthogonal set over the unit circle, which means that they do not form a complete base of polynomials (i. e., they are not linearly independent). In 1934 the Dutch physicist Fritz Zernike published an article containing a different set of polynomials for description of optical aberrations [2]. These polynomials have certain mathematical properties, such as orthogonality over the unit circle and invariance with rotation, which make them specially interesting for wave-front fitting. Because of the simple mathematics involved to generate these sets of polynomials at any desired order, highly precise fitting of the aberration function could be accomplished. These polynomials became gradually popular among optical scientists in the second half of the $20^{t h}$ century and are widely used today in many different fields of optics, including vision optics and optical engineering. They became commonly known as Zernike Polynomials. We have chosen to use these polynomials instead of those of Seidel in the present work, for the above reasons and also because they have been chosen as the conventional polynomials for aberration description in vision science by a special comity of the Optical Society of America [3] . A formal mathematical presentation of Zernike Polynomials and their equations and properties is given in section (3).

\section{I.2 Aberration in vision optics}

Until the mid 80s there were only two options for the ophthalmologist in order to correct ametropia: spectacles or contact lenses. Of course, for both these techniques, the main objective was to correct the three lower order ametropia. There was no motivation for correction of higher order aberrations for two basic reasons: (1) there was no instrumentation for measuring them and, even if one could make more precise measurements, (2) there were no power lathe that could possibly mold optical surfaces with profiles more sophisticated than toroidal surfaces (the summation of a spherical plus a cylindrical surface).

In this scenario, there was no apparent need for better instruments than the conventional refractometers, developed in the early 70 s $[4,5]$. After the advent of computerized and high precision instruments for corneal surface measurements (usually referred to as Corneal Topographers)[6] in the mid $80 \mathrm{~s}$ and pulsed dye lasers for molding the cornea into elaborate forms in the early 90s [7], the need for more precise refractometers became evident. The necessity for such instrumentation started to be addressed in 1994 when Liang and colleagues [8] suggested, for the first time, the 
application of the HS sensor $[9,10]$ for measuring the aberrations of the in vivo eye. This was a truely innovative idea given the fact that HS sensor were popular in other optical fields at the time, but had never been applied to the human eye. It became clear that, if vision correction is to achieve a "state of the art" status, higher order aberrations should be measured and corrected, much in the same that this correction was already happening at high magnification telescopes since the early 1950s [11].
In the next section we present the second generation of an instrument that has been developed at Lab. de Óptica Oftálmica - IFSC. The first generation prototype has presented partially elsewhere [12].

\section{Instrumentation}

The diagram of Fig. 2 shows a simple scheme of our instrument.

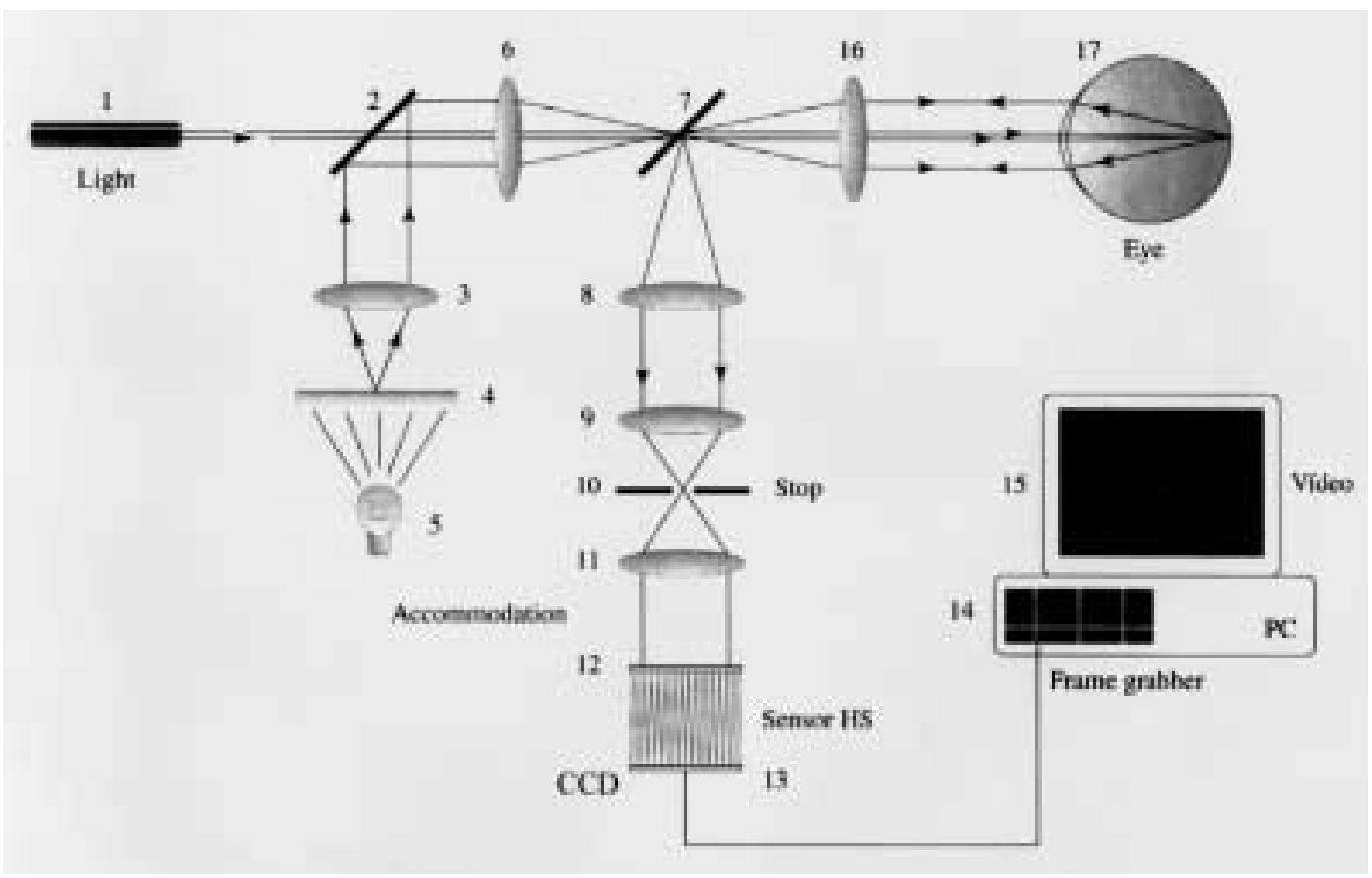

Figure 2. Schematic diagram of optical setup used to measure aberrations of the mechanical eye.

A He-Ne laser beam (1) is focused at the back of the eye. In this first optical path the goal is to generate a small spot of light at the retina, by adjusting position of lens (16). The accommodation system consists of a light bulb (5) that shines a picture (5), which is viewed by the eye. Lens (3) is shifted until the far point of the eye is found. The diffused light reflected at the retina return passing by all eye components (vitreous humor, crystalline, aqueous humor, cornea), goes through lens (16), reflects on the beam splitter (7) and continuous through lenses (8), (9) and (11), going through the stop (10). The stop eliminates reflections from the accommodation system, from the cornea and lens (16). Finally the wave-front hits at the HS sensor (12) and is focused at the CCD array (13). The CCD image is digitized in a "frame grabber" (14) and processed at an IBM PC, which displays the graphical information at the colored monitor (15).

In Fig. 3 we may see an actual photograph of the instrument mounted at the Lab. de Óptica Oftálmica - IFSC. The components shown in Fig. 3 are in accordance to the optical diagram shown in Fig. 2. The equipment was mounted over an moveable aluminum base with a commercial head and chin rest was adapted to it. The accommodation system is still under development [13] for future tests on in vivo eyes.

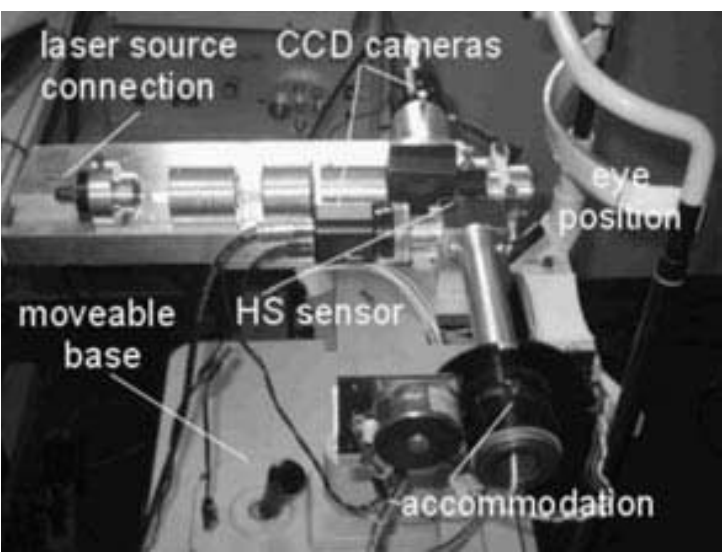

Figure 3. Wave-front instrument developed at the IFSC. 
The working principle of the HS sensor is depicted in Fig. 4. In Fig. 4(a) we present HS principle for a plane wave-front and a distorted wave-front, respectively. For a plane wave front the focusing position on the CCD plane is exactly at the intersection of the axis of the micro-lens with the CCD $x, y$ plane; on the other hand, for an aberrated wave-front, light focuses at a spot slightly shifted. For a set of micro-lenses (Fig. 4(b)) the same principle applies and we will have uniformly spaced spots for a plane wave and non-uniformly distributed points for aberrated waves. The amount of displacement of the spots in each direction $(\Delta x, \Delta y)$ is what allows us to determine the exact amount of wave-front aberration. This procedure is described in the following section.

(a)

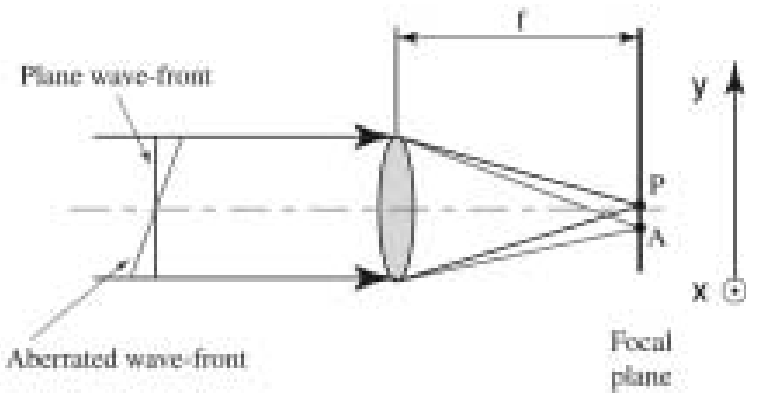

(b)

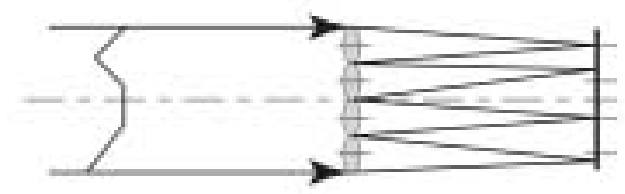

Aberracel tunulle of rays

Figure 4. Principle of the HS sensor, showing plane (a) and (b) distorted wave-fronts hitting the CCD sensor.

\section{Representing optical aberrations with Zernike Polynomials}

Zernike polynomials are a set of polynomial maps from the unit disc $x^{2}+y^{2} \leq 1$ of the $x, y$-plane into the $z$-axis, which have the desirable property that combinations of these maps can be found to well fit the surface shapes of wave-front aberrations. In polar coordinates, Zernike polynomials are the product of a radial polynomial and a azimuthal map:

$$
Z_{n}^{l}(\rho, \theta)=\{R(\rho) \cos l \theta \text { if } l<0 R(\rho) \sin l \theta \text { if } l \geq 0
$$

where $l$ may be any integer number and $n$ may be any positive integer and zero. When $l$ is greater than or equal to zero, the sine function is used and when it is smaller than zero the cosine function is used. The radial components of the Zernike polynomials are given by:

$$
R_{n}^{l}(\rho)=\sum_{s=0}^{(n-l) / 2} \frac{(-1)^{s}(n-s) !}{s ![(n+m) / 2-s] ![(n-m) / 2-s] !} \rho^{n-2 s}
$$

The Zernike polynomials are thus a set of orthogonal maps defined in the unit circle. Their orthogonality condition is expressed by:

$$
\int_{0}^{1} \int_{0}^{2 \pi} Z_{n}^{l}(\rho, \theta) Z_{n^{\prime}}^{l^{\prime}}(\rho, \theta) \rho d \rho d \theta=\frac{\pi}{2(n+1)} \delta_{n n^{\prime}} \delta_{l l^{\prime}}
$$

The main motivation for using Zernike polynomials is that they describe with high precision the shapes of four conventional Seidel aberrations. Because there is no limit on the number of terms that may be used, many higher order aberrations can be described by Zernike polynomials, among them coma, $3^{\text {rd }}$ order spherical aberration, etc.. Actually it is enough to use the first 15 linearly independent Zernike polynomials (see Table 2), in order to obtain a highly accurate description of the most common aberrations found in human eyes. These polynomials, in mathematical terms, comprise a set of linearly independent polynomials in two indeterminates with degree less than or equal to 4 , which are orthonormal with respect to the inner product given in (7).

We mention in section (3) the possibility of calculating the wave-front aberration from the wave-front slopes. Based on figure 4 we may write the slopes in the $x$ and $y$ directions as:

$$
\begin{aligned}
& W_{x}^{\prime}=\frac{\Delta x}{f}=\frac{x_{a}-x_{c}}{f} \\
& W_{y}^{\prime}=\frac{\Delta y}{f}=\frac{y_{a}-y_{c}}{f}
\end{aligned}
$$

where $\left(x_{a}, y_{a}\right)$ is the coordinate of a spot from an aberrated wave-front, $\left(x_{c}, y_{c}\right)$ is the coordinate of the reference spot, i. e., a non-aberrated, plane wave-front, and $f$ is the focal length of the micro-lenses. If we use a single index $k$ as function of $n$ and $l$ ( the indexes of equations (3)-(5)), namely,

$$
k=\frac{n(n+1)}{2}+\frac{n-l}{2}+1
$$

then we may write the wave-front as:

$$
W(x, y)=\sum_{k=1}^{15} C_{k} Z_{k}(x, y)
$$

and its partial derivatives as

$$
\begin{gathered}
W_{x}^{\prime}(x, y)=\sum_{k=1}^{15} C_{k} \frac{d Z_{k}(x, y)}{d x} \\
W_{y}^{\prime}(x, y)=\sum_{k=1}^{15} C_{k} \frac{d Z_{k}(x, y)}{d y} .
\end{gathered}
$$

Note that $k$ is the number of the Zernike term in accordance to Table 2. In order to find the Zernike coefficients for a specific wave-front, we perform a minimum square fit for all $i, j$ centroids which form the HS image. This procedure consists of minimizing the sum 


$$
S=\sum_{i, j=1}^{n}\left[\left(W_{x}^{\prime}\left(x_{i j}, y_{i j}\right)-\frac{d Z}{d x}\left(x_{i j}, y_{i j}\right)\right)^{2}+\left(W_{x}^{\prime}\left(x_{i j}, y_{i j}\right)-\frac{d Z}{d x}\left(x_{i j}, y_{i j}\right)\right)^{2}\right]
$$

relative to each Zernike coefficient, therefore we have to find $\frac{d S}{d C_{t}}=0$ for $t=1, \ldots, k$

$$
\begin{gathered}
\frac{d S}{d C_{t}}=\sum_{i, j=1}^{n}\left(W_{x}^{\prime}\left(x_{i j}, y_{i j}\right) \frac{d Z_{t}}{d x}\left(x_{i j}, y_{i j}\right)-W_{y}^{\prime}\left(x_{i j}, y_{i j}\right) \frac{d Z_{t}}{d y}\left(x_{i}, y_{i}\right)-\right. \\
\sum_{k=1}^{l} C_{k} \sum_{i, j=1}^{n}\left(\frac{d Z_{k}}{d x}\left(x_{i j}, y_{i j}\right) \frac{d Z_{t}}{d x}\left(x_{i j}, y_{i j}\right)+\frac{d Z_{k}}{d y}\left(x_{i j}, y_{i j}\right) \frac{d Z_{t}}{d y}\left(x_{i j}, y_{i j}\right)=0\right.
\end{gathered}
$$

from where we extract a square linear system $A C=b$ with $k$ equations and $k$ unknown values of $C$. By solving this linear system through conventional procedures ( $L U$ decomposition and Gaussian elimination method) [14] we find 15 values of $C$ for each measured eye. For a flat wave-front the partial derivatives $\frac{d W}{d x}$ and $\frac{d W}{d y}$ are zero, and we therefore obtain a solution $C=0$, which by the general equation (11) renders $W\left(x_{i j}, y_{i j}\right)=0$, a plane wave in the $x y$ plane. Now for wave-fronts that are not flat we obtain nonzero values of partial derivatives, therefore the linear system will not have a trivial solution. The coefficients that contribute the most to a specific aberration will have greater values. For example, if the measured eye has a great amount of coma the eighth and ninth coefficient will have values greater than others. Otherwise if there is a greater amount of astigmatism at $45^{\circ}$, the fourth term will predominate. In the next section we show results of calculation for several calibrated aberrations for a mechanical eye.

\section{Preliminary Results}

Measurements were made in a mechanical (artificial) eye which was calibrated with five different ametropia: emmetropic (0D), hyperopic (+5D), myopic (-5D), and 2D astigmatism at $45^{\circ}$ and $90^{\circ}$ for the cylinder axis (observation: in ophthalmology the quantification of an ametropia is usually given by the spherical plus cylindrical components of a toric lens). An image processing algorithm to find the HS spots was implemented in Pascal (Borland Delphi version 5.0) using conventional Computer Vision techniques such as segmentation and filtering $[15,16]$. Image processing was accomplished separately for each aberration (see Fig. 5, column A) and results were plotted in several different outputs.

In the Fig. 5, from left to right column, respectively, we may see results for the image processing (A), twodimensional color coded maps for the wave-front (B), surface maps (C) and, finally, the Zernike coefficients for each of the 15 terms in Table 2 (D). A qualitative analysis for the first row (0D) shows regular spaced dots, a color map with one color, a plane of hight one, and coefficients all with value zero. This is obviously in accordance with the expected values for a zero dioptric eye, i.e., with no aberrations, therefore resulting in a plane wave leaving the eye. The same qualitative analysis for the other eyes show the validity of results. For example, in the cases of $+5 \mathrm{D}$ and $-5 \mathrm{D}$, we know that the wave-front should look like a dome shaped surface, facing downwards for the myopic (strong optical system $=$ converging wave-front) and upwards for the hyperopic (weaker optical system $=$ diverging wave-front). This is in accordance to the expected shapes of wave-fronts leaving eyes with different low order aberrations.

It is possible to relate the wave-front measurements with those of refractometers. Regardless of the wave-front's typical high resolution data, it is still possible to retrieve conventional dioptric power values for the best spherocylinder lens that describes the eye's lower order aberrations. If we consider the spherocylindrical lens as

$$
W_{\text {lens }}=2 C_{4} x y+2 C_{5}\left(x^{2}+y^{2}\right)+C_{6}\left(y^{2}-x^{2}\right)
$$




\begin{tabular}{|c|c|c|c|}
\hline Term & Polar & Cartesian & Meaning \\
\hline$Z_{1}(x, y)$ & 1 & 1 & Constant term \\
\hline$Z_{2}(x, y)$ & $\rho \sin \theta$ & $x$ & $\begin{array}{l}\text { Tilt in } x \\
\text { direction }\end{array}$ \\
\hline$Z_{3}(x, y)$ & $\rho \cos \theta$ & $y$ & $\begin{array}{l}\text { Tilt in } y \\
\text { direction }\end{array}$ \\
\hline$Z_{4}(x, y)$ & $\rho^{2} \sin (2 \theta)$ & $2 x y$ & $\begin{array}{l}\text { Astigmatism } \\
\text { with axis at } \\
\pm 45^{\circ}\end{array}$ \\
\hline$Z_{5}(x, y)$ & $2 \rho^{2}-1$ & $-1+2 y^{2}+2 x^{2}$ & Focus shift \\
\hline$Z_{6}(x, y)$ & $\rho^{2} \cos (2 \theta)$ & $y^{2}-x^{2}$ & $\begin{array}{l}\text { Astigmatism } \\
\text { with axis at } 0 \\
\text { or } 90^{\circ}\end{array}$ \\
\hline$Z_{7}(x, y)$ & $\rho^{3} \sin (3 \theta)$ & $3 x y^{2}-x^{3}$ & \\
\hline$Z_{8}(x, y)$ & $\left(3 \rho^{3}-2 \rho\right) \sin \theta$ & $-2 x+3 x y^{2}+3 x^{3}$ & $\begin{array}{l}\text { Third order } \\
\text { coma along } x \\
\text { axis }\end{array}$ \\
\hline$Z_{9}(x, y)$ & $\left(3 \rho^{3}-2 \rho\right) \cos \theta$ & $-2 y+3 x^{2} y+3 y^{3}$ & $\begin{array}{l}\text { Third order } \\
\text { coma along } y \\
\text { axis }\end{array}$ \\
\hline$Z_{10}(x, y)$ & $\rho^{3} \cos (3 \theta)$ & $y^{3}-3 x^{2} y$ & \\
\hline$Z_{11}(x, y)$ & $\rho^{4} \sin (4 \theta)$ & $4 y^{3} x-4 x^{3} y$ & \\
\hline$Z_{12}(x, y)$ & $\left(4 \rho^{4}-3 \rho^{2}\right) \sin (2 \theta)$ & $-6 x y+8 y^{3} x+8 x^{3} y$ & \\
\hline$Z_{13}(x, y)$ & $6 \rho^{4}-6 \rho^{2}+1$ & $1-6 y^{2}-6 x^{2}+6 y^{4}+12 x^{2} y^{2}+6 x^{4}$ & $\begin{array}{l}\text { Third or- } \\
\text { der spherical } \\
\text { aberration }\end{array}$ \\
\hline$Z_{14}(x, y)$ & $\left(4 \rho^{4}-3 \rho^{2}\right) \cos (2 \theta)$ & $-3 y^{2}+3 x^{2}+4 y^{4}-4 x^{2} y^{2}-4 x^{4}$ & \\
\hline$Z_{15}(x, y)$ & $\rho^{4} \cos (4 \theta)$ & $y^{4}-6 x^{2} y^{2}+x^{4}$ & \\
\hline
\end{tabular}

Table 2. Set of first 15 Zernike polynomials

the sphere $\left(\phi_{D}\right)$, cylinder $\left(\phi_{A}\right)$ and axis $(\alpha)$ may be written as:

$$
\begin{gathered}
\phi_{A}=4 \sqrt{6} \frac{\sqrt{a_{4}^{2}+a_{6}^{2}}}{r^{2}} \\
\alpha= \begin{cases}90+\arctan \left(\frac{C_{6}}{C_{4}}\right) & \text { for } \frac{C_{6}}{C_{4}}>0 \\
\arctan \left(\frac{C_{6}}{C_{4}}\right) & \text { for } \frac{C_{6}}{C_{4}} \leq 0\end{cases} \\
\phi_{D}=\frac{4 \sqrt{C_{5}}}{d^{2}}
\end{gathered}
$$

where $d$ is the diameter of the entrance pupil.

The RMSE for these measurements were as follows: $0.04 \mathrm{D}$ for sphere and cylinder and $4^{0}$ for axis. It is known that auto-refractors have typically errors of $0.12 \mathrm{D}$ diopters for sphere and cylinder and $5^{0}$. As we may see from these preliminary measurements the wave-front device allows for more precise exams. We believe this precision is reproducible for eyes of the general population.

\section{Discussion}

We have demonstrated the precision the HS wave-front sensor in measuring optical aberrations of a calibrated mechanical eye. Tests on in vivo eyes should be conducted for verification and validation of the technique.

One common difficulty of instruments when attempting to measure refractive errors happens during accommodation. It is not always possible to repeat the measurements with the crystalline lens at exactly the same power. This certainly makes the reproductivity of these instruments much lower than expected. Our intention here was not to compare precision of the whole refractive instrumentation, therefore we did not consider accommodation (our artificial eyes do not have a lens). Furthermore our intention was to provide insight to how promising the wave-front technique may be when compared to conventional auto-refractors, in terms of resolution and precision. An accommodation system, when in well functioning condition, may be applied to any of both instruments and than absolute precision comparisons for daily measurements in the human population may be undertaken. 

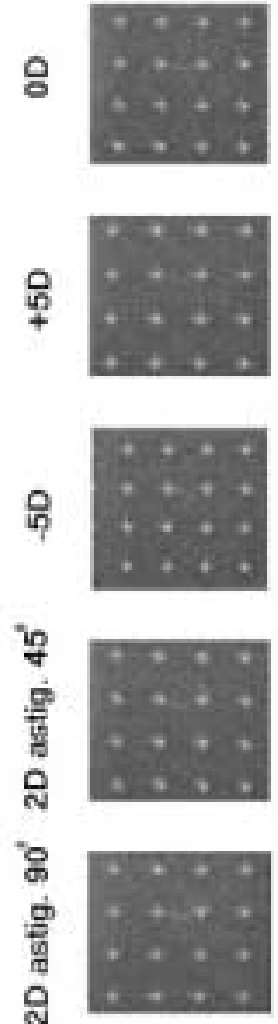

(A)
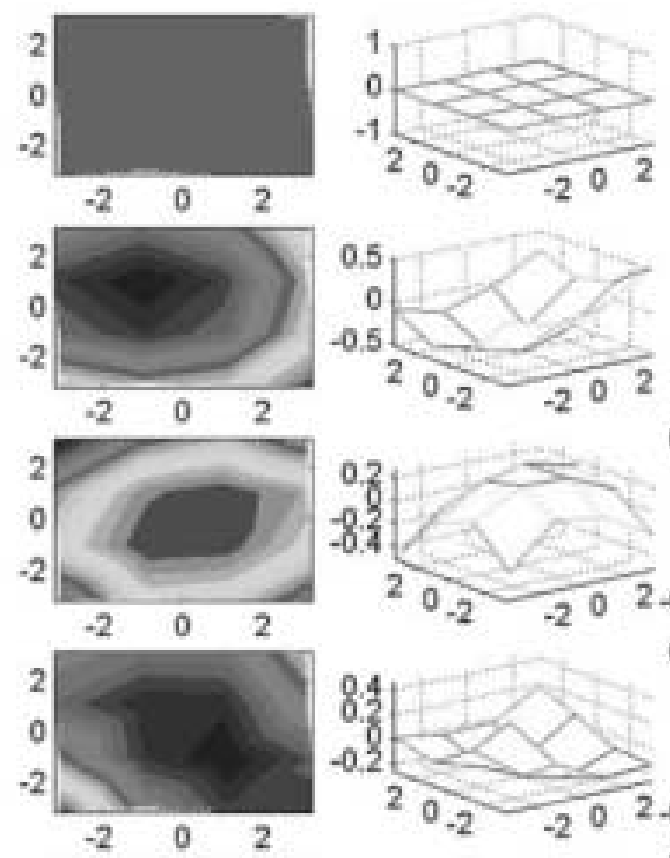

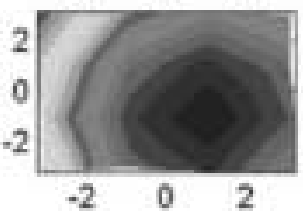

(B)

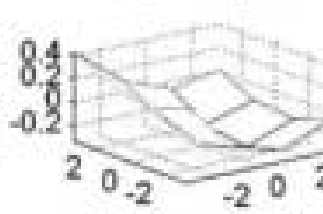

(C)
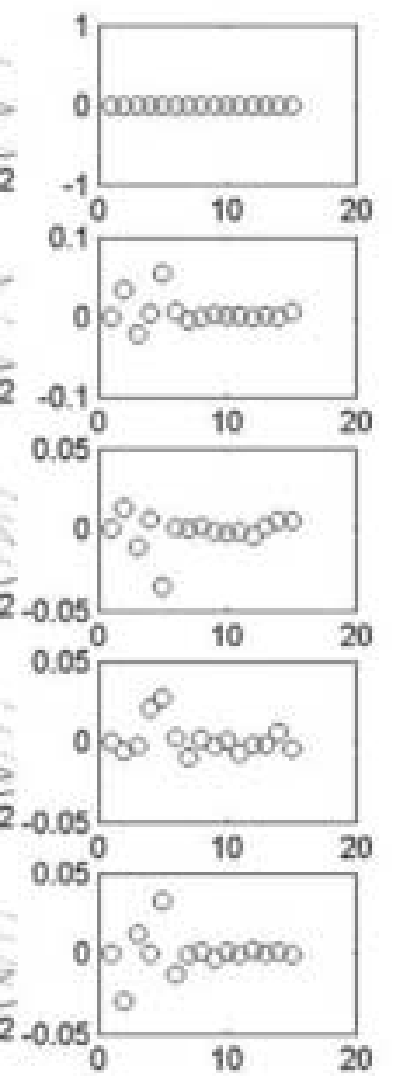

(D)

Figure 5. Wave-front results for all 5 cases.

An important factor is the amount of micro-lenses in the HS sensor. If we double the number of micro-lenses in the row and column, the resolution is multiplied by a factor of 4 . On the other hand, for highly distorted corneas this might be a disadvantage. In a previous work $[17,18]$ we have shown, by implementing computer simulations of HS patterns for several corneal topographies, that for eyes with severe curvature changes on corneal surface (a pathology commonly known as keratoconus), the HS spots may overlap, preventing the software from the capacity of image processing and data analysis. In these specific cases, conventional trial lens tests with auto-projectors or Snellen tables may still be necessary.

We strongly believe that refractometers based on the HS technology represent the next generation of high resolution refraction instruments and that they will gradually be available in most eye-care clinics and hospitals, much in the same way that HS sensors have also become wide-spread in high magnification astronomical telescopes throughout the world. Moreover, the high resolution wave-front data will allow for precise corneal surgeries [19, 20, 21], resulting in algorithms that may execute what is being called "customized corneal ablations" [22].

Acknowledgements This research was partially supported by Fapesp - grant \# 01/03132-8. We would also like to thank Dr. Wallace Chamon, Dr. Paulo Schor and Dr. Rubens Belfort Jr., from the Escola Paulista de Medicina - UNIFESP for their valuable insights.

\section{References}

[1] M. Born, Principles of Optics Pergamon Press, 1975: 464466.

[2] F. Zernike, Beugungstheorie des Schneidenverfahrans und Seiner Verbesserten Form, der Phasekontrastmethode, Physica $1,1943$.

[3] L.N. Thibos, R.A. Applegate, J.T. Schwiegerling, R. Webb, and VSIA Standards Taskforce Members, Standards for reporting the optical aberrations of eyes, Opt Soc Am, Visual Science and Applications, 1999.

[4] W.N. Charman, A pioneering instrument: the Collin's electronic refractionometer, Opthalmol Opt, 16, 345 (1976).

[5] T.N. Cornsweet, H.D. Crane, Servo-controlled infrared optometer, J Opt Soc Am, 60, 1 (1976).

[6] S.D. Klyce, Computer-Assisted Corneal Topography, High Resolution Graphics Presentation and Analyses of Keratoscopy, Invest. Ofthalmol Vis Sci, 25, 426 (1984). 
[7] A. Unkroth, J. Kleinschmidt, W. Ziegler, B. Hofmann, Jutte M. Graefes, Ablation of the cornea by using a low-energy excimer laser, Arch Clin Exp Ophthalmol 231(5), 303 (1993).

[8] J. Liang, B. Grimm, S. Goelz, J.F. Bille, Objective measurement of wave aberrations of the human eye with the use of a Hartmann-Shack wave-front sensor, J Opt Soc Am 14, 1949 (1994).

[9] J. Hartmann, Bemerkungen uber den bau und Die Justirung von Spektrographen, Z Instrumentenkd, 2047 (1900).

[10] R.V. Shack, B.C. Platt, Production and use of a lenticular Hartmann screen, Optical Sciences Center, University of Arizona, Tucson, Spring Meeting, Optical Society of America, 1971: 656.

[11] H.W. Babcock, The possibility of compensating astronomical seeing, Publ Astron Soc Pac, 56, 229 (1953).

[12] F.S. Junior, F. Yasuoka, J. Santos, L.A. Carvalho, J.C. Castro, Desenvolvimento de um sistema para medidas de aberrações ópticas do olho humano usando a técnica de HartmannShack, XXIV Encontro de Física da Matéria Condensada, São Lourenço - MG, Fevereiro de 2001.

[13] Francisco A. Scannavino Jr., Romeu L. Filho, Jesulino B. Santos, Luis A.V. Carvalho, Jarbas C. Castro N., Antônio C. Romão, Instrumento Eletro-óptico para medidas automatizadas da acomodação do olho humano (IN VIVO) durante medidas refrativas, XXIV Encontro Nacional de Física da Matéria Condensada, 2001, PG. 458.

[14] W.H. Press, B.P. Flannery, S.A. Teukolsky, W.T. Vetterling, Numerical recipes in pascal - the art of scientific computing, Cambridge University Press, 1989, pag. 37-39.

[15] R.C. Gonzalez, R.E. Woods, Digital image processing, Addison Wesley, 1992.
[16] L.A. Carvalho, M. Stefani, A.C. Romão, S. Tonissi, J. Castro, Digital Processing of image reflectd from the Lachrymal Film of the Anterior Corneal Surface, Revista Brasileira de Engenharia Biomdica, 17113 (2001).

[17] L.A. Carvalho, J.C. Castro, P. Schor, W, Chamon, A software simulation of Hartmann-Schack patterns for real corneas, International Symposium: Adaptive Optics: from telescopes to the human eye, Murcia, Spain, November 13-14, 2000.

[18] Luis Alberto Vieira de Carvalho, Jarbas Caiado de Castro, Wallace Chamon, Paulo Schor, Luis Antonio Vieira de Carvalho, Wave-front measurements of the human eye using the Hartmann-Shack sensor and current state-of-the-art technology for excimer laser refractive surgery, Lasik and Beyond Lasik, Section V, Chapter 31, Highlights of Ophthalmology, 2001.

[19] P.R. Krueger, In perspective: Eye tracking and Autonomous laser radar, J Refract Surg 2, 145 (1999).

[20] G.H. Pettit, J.A. Campin, B.J. Housand, K.K. Liedel, Customized corneal ablation: wavefront guided laser vision correction, Autonomous Technology (Orlando, Fl) ARVO (The Association for Research in Vision and Ophthalmology), Annual Meeting, Fort Lauderdale, Florida (USA), May 914,1999 .

[21] J.A. Campin, G.H. Pettit, G.P. Gray, Required laser beam resolution and PRK system configuration for custom high fidelity corneal shaping, Invest Ophthalmol Vis Sci 1999; 38(suppl): S538.

[22] S.A. Klein, Optimal corneal ablation for eyes with arbitrary Hartmann-Shack aberrations, J Opt Soc Am Vol 15, 2580 (1998). 